Rev. Adm. Saúde - Vol. 18, № 70, jan. - mar. 2018

http://dx.doi.org/10.23973/ras.70.84

ARTIGO ORIGINAL

\title{
Gestão de riscos e segurança do paciente: mapeamento dos riscos de eventos adversos na emergência de um hospital de ensino
}

Risk management and patient safety: mapping the risk of adverse events in the emergency of a teaching hospital

\section{Maria Girlane Sousa Albuquerque Brandão1, Odézio Damasceno Brito², Lívia Moreira Barros ${ }^{3}$}

1. Acadêmica de enfermagem do 9ํㅗㄴ semestre pela Universidade Estadual Vale do Acaraú, Sobral CE

2. Enfermeiro, residente em urgência e emergência na Universidade Estadual Vale do Acaraú, Sobral CE

3. Doutora em enfermagem. Docente da Universidade Estadual Vale do Acaraú, Sobral CE

\section{RESUMO}

Este estudo tem por objetivo identificar o risco de desenvolvimento de eventos adversos na emergência de um hospital de ensino. Trata-se de uma pesquisa descritiva, transversal e quantitativa, desenvolvida na emergência de um hospital de ensino público no estado do Ceará. A população investigada constituiu-se de 163 pacientes e a coleta de dados foi realizada diariamente durante os três turnos de domingo a domingo durante o mês de julho de 2017, por observação participante e entrevista com os pacientes a partir de instrumento estruturado. Com relação aos dados sociodemográficos verificouse a predominância de pacientes do sexo masculino (65\%). A idade variou de 18 a 94 anos, com predominância na faixa etária de 50 a 69 anos (32,5\%). No tocante aos riscos para eventos adversos, foram nomeados os quatro tipos predominantes quanto ao risco de se desenvolverem na unidade em estudo: risco de flebite $(82,8 \%)$, risco de quedas $(26,9 \%)$, risco de broncoaspiração $(39,2 \%)$ e risco de lesões por pressão $(58,8 \%)$. Este estudo permitiu concluir 
que a identificação de riscos é fundamental para a prevenção de eventos adversos nos serviços hospitalares, sendo uma prática importante para qualificar a assistência e impedir que o paciente desenvolva algum dano durante o período intra-hospitalar.

Palavras-chave: serviço hospitalar de emergência, segurança do paciente, cuidados de enfermagem, qualidade da assistência à saúde.

\section{ABSTRACT}

This study aims to identify the risk of developing adverse events in the emergence of a teaching hospital. This is a descriptive, cross-sectional and quantitative research, developed in the emergence of a public teaching hospital in the state of Ceará. The study population consisted of 163 patients and data collection was performed daily during the three shifts from Sunday to Sunday during the month of July 2017, by participant observation and interview with the patients from a structured instrument. Regarding the socio-demographic data, the predominance of male patients (65\%) was found. Age ranged from 18 to 94 years, predominantly in the age range of 50 to 69 years $(32,5 \%)$. Concerning the risks for adverse events, the four predominant types were considered for risk of developing in the study unit: risk of phlebitis (82,8\%), risk of falls $(26,9 \%)$, risk of bronchoaspiration $(39,2 \%)$ and risk of pressure injuries $(58,8 \%)$. This study allowed us to conclude that the identification of risks is fundamental for the prevention of adverse events in hospital services, being an important practice to qualify care and prevent the patient from developing any damage during the in-hospital period.

Keywords: emergency hospital service, patient safety, nursing care, quality of health care.

\section{INTRODUÇÃO}

Nos tempos atuais, a assistência à saúde perpassa não apenas por aspectos técnico-científicos, mas também por um conjunto de dimensões que explicitam a cultura organizacional de cada serviço de saúde, em particular de cada organização hospitalar. O hospital é considerado, entre as organizações existentes, uma das mais complexas, cujo funcionamento se dá por meio da interação entre os setores envolvidos e a necessidade de harmonizar os processos operacionais, em outras palavras, desenvolver ações que promovam, com responsabilidade, qualidade e segurança no atendimento ao paciente que procurou o serviço em busca de bem-estar e saúde. ${ }^{1}$

A segurança do paciente pode ser compreendida como a redução, a um mínimo aceitável, do risco de dano desnecessário associado ao cuidado de 
saúde. ${ }^{2}$ Estudos estimam que a ocorrência de incidentes relacionados à assistência à saúde, e em particular os eventos adversos, afete de $4 \%$ a $16 \%$ de pacientes hospitalizados em países desenvolvidos, o que sensibilizou sistemas de saúde em todo o mundo a melhorar a segurança do paciente. ${ }^{3}$

Uma vez que, é direito de todo e qualquer indivíduo, receber uma assistência à saúde de qualidade e livre de danos, e dever de todo serviço de saúde prestar uma assistência eficiente e segura em todos os seus processos. ${ }^{4}$

A segurança do paciente deve ser valorizada, mais do que nunca, principalmente nos hospitais, onde os profissionais trabalham em busca do melhor para os pacientes, mas que, devido à elevada complexidade, estes podem estar em risco só pelo fato de lá se encontrarem. Nos hospitais, a chance de o erro acontecer é elevada pelo fato de a prestação de serviços estar ligada a complexas interações entre pessoas, instalações, equipamentos e medicamentos. ${ }^{5}$

Nas últimas décadas, diante de vários estudos evidenciarem a ocorrência de eventos adversos nas instituições de saúde ao redor do mundo, a preocupação com a segurança do paciente tornou-se assunto prioritário na área da saúde. Assim, o Programa Nacional de Segurança do Paciente (PNSP), instituído no Brasil pela Portaria MS oㅡ 529, de 1 de abril de 2013, demonstra comprometimento governamental contribuindo para a qualificação do cuidado em saúde em todos os estabelecimentos de saúde do território nacional, e promovendo maior segurança para pacientes, profissionais de saúde e ambiente de assistência à saúde. ${ }^{6}$

Entendendo que a qualidade da assistência influencia, de forma direta, para uma boa evolução do estado de saúde dos pacientes e que essa qualidade está intimamente relacionada com a segurança da assistência prestada pela equipe de enfermagem, pretende-se com o presente estudo responder às seguintes indagações: quais eventos adversos estão presentes na emergência de um hospital de ensino da região norte do Ceará? Qual o risco de tais eventos adversos acontecerem nos pacientes admitidos no setor de emergência hospitalar?

Diante dessas considerações, estudar a cultura de segurança no hospital traz a possibilidade de conhecer os fatores intervenientes no processo de trabalho que impactam na segurança dos pacientes. $O$ desenvolvimento de pesquisas na área permite discutir essa cultura e a apreensão desse conhecimento leva a fortalecer a comunicação efetiva de evidências científicas, experiências e recomendações destinadas a garantir a segurança dos pacientes na atenção à saúde. ${ }^{7}$

Nessa reflexão é que este estudo foi desenvolvido, tendo por objetivo identificar os riscos de eventos adversos na emergência de um hospital de ensino.

\section{MATERIAL E MÉTODOS}


Trata-se de uma pesquisa descritiva, transversal, com abordagem quantitativa, desenvolvida em um hospital de ensino público do interior do estado do Ceará, o qual conta com capacidade operacional total de 411 leitos conveniados ao Sistema Único de Saúde (SUS), bem como atende uma população de aproximadamente dois milhões de habitantes e faz uma média anual de mais de 22 mil internações.

O estudo foi realizado na unidade de emergência adulta do referido hospital, que conta com 55 leitos. A população investigada constituiu-se de 163 pacientes e a coleta de dados foi realizada diariamente durante os três turnos (matutino, vespertino e noturno) de domingo a domingo durante o mês de julho de 2017, por observação participante e entrevista com os pacientes internados a partir de instrumento estruturado. Acrescentou-se ao referido instrumento, variáveis que contemplavam a caracterização sociodemográfica.

Para definição da quantidade de participantes, foram considerados os seguintes critérios de inclusão: possuir idade superior a 18 anos e pacientes que foram classificados quanto ao seu risco de saúde no acolhimento.

Os dados obtidos foram tabulados no programa Excel 2016 e analisados no programa estatístico Statistical Package for the Social Sciences (SPSS), versão 20, para Windows, e armazenados em banco de dados, no qual os resultados estão apresentados com frequências absolutas e relativas em tabelas.

Cumpre salientar que todas as exigências éticas dispostas na Resolução 466/2012 do Conselho Nacional de Saúde foram integralmente respeitadas. Ademais, o projeto de pesquisa que fomentou este estudo foi submetido e aprovado pelo Comitê de Ética em Pesquisa da Universidade Estadual Vale do Acaraú (CAAE: 68436717.3.0000.5053) e sua realização foi autorizada pela instituição em estudo.

Obteve-se a dispensa da assinatura do Termo de Consentimento Livre e Esclarecido (TCLE), pois a avaliação das demandas de cuidado junto ao paciente é considerada uma atividade cotidiana do enfermeiro e os pacientes não foram submetidos a nenhum outro procedimento em função da aplicação do instrumento.

\section{RESULTADOS}

Foram incluídos no estudo 163 pacientes. Com relação aos dados sociodemográficos (Tabela 1) verificou-se a predominância de pacientes do sexo masculino (65\%). A idade variou de 18 a 94 anos, com predominância na faixa etária de 50 a 69 anos. No que diz respeito aos aspectos clínicos, o tempo de internação alternou de um a 29 dias, com média de 3,3 dias $( \pm 3,2)$. Em relação à procedência, $78,5 \%$ dos pacientes advieram de cidades circunvizinhas, e $21,4 \%$ dos pacientes internados na emergência tiveram procedência da cidade de Sobral. 
Tabela 1. Perfil dos pacientes admitidos na emergência hospitalar.

\begin{tabular}{llrr}
\hline & & $\mathbf{N}$ & $\%$ \\
\hline Sexo & Maculino & 106 & 65,0 \\
\cline { 2 - 4 } & Feminino & 57 & 35,0 \\
\hline Faixa etária & 18 a 29 anos & 16 & 9,8 \\
\cline { 2 - 4 } & 30 a 49 anos & 52 & 31,9 \\
\cline { 2 - 4 } & 50 a 69 anos & 53 & 32,5 \\
\cline { 2 - 4 } & 70 a 89 anos & 04 & 23,3 \\
\cline { 2 - 4 } & $>90$ anos & 35 & 21,4 \\
\hline Procedência & Sobral & 128 & 78,6 \\
\cline { 2 - 4 } & Cidades circunvizinhas & & 2,4 \\
\hline
\end{tabular}

No que tange ao acolhimento com classificação de risco (Gráfico 1), 53,3\% tiveram classificação de risco amarela. Quanto à internação, 51 pacientes com casos neurológicos, 54 casos clínicos e 58 traumatológicos.

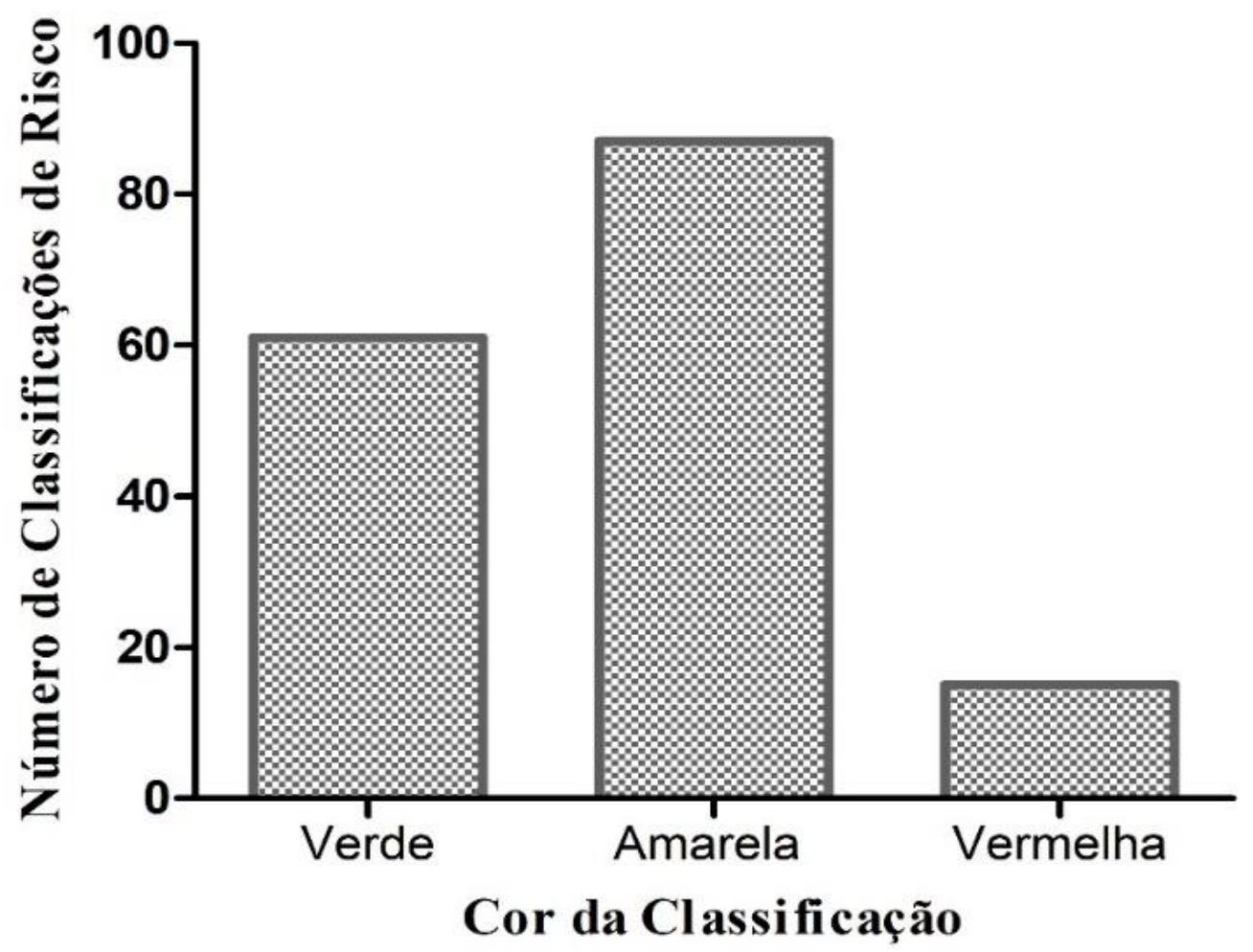


Gráfico 1. Distribuição da classificação de risco em uma unidade de emergência.

No tocante aos riscos para eventos adversos (Gráfico 2), foram nomeados os quatro tipos predominantes quanto ao risco de se desenvolverem na unidade em estudo: risco de flebite, risco de quedas, risco de broncoaspiração e risco de lesões por pressão.

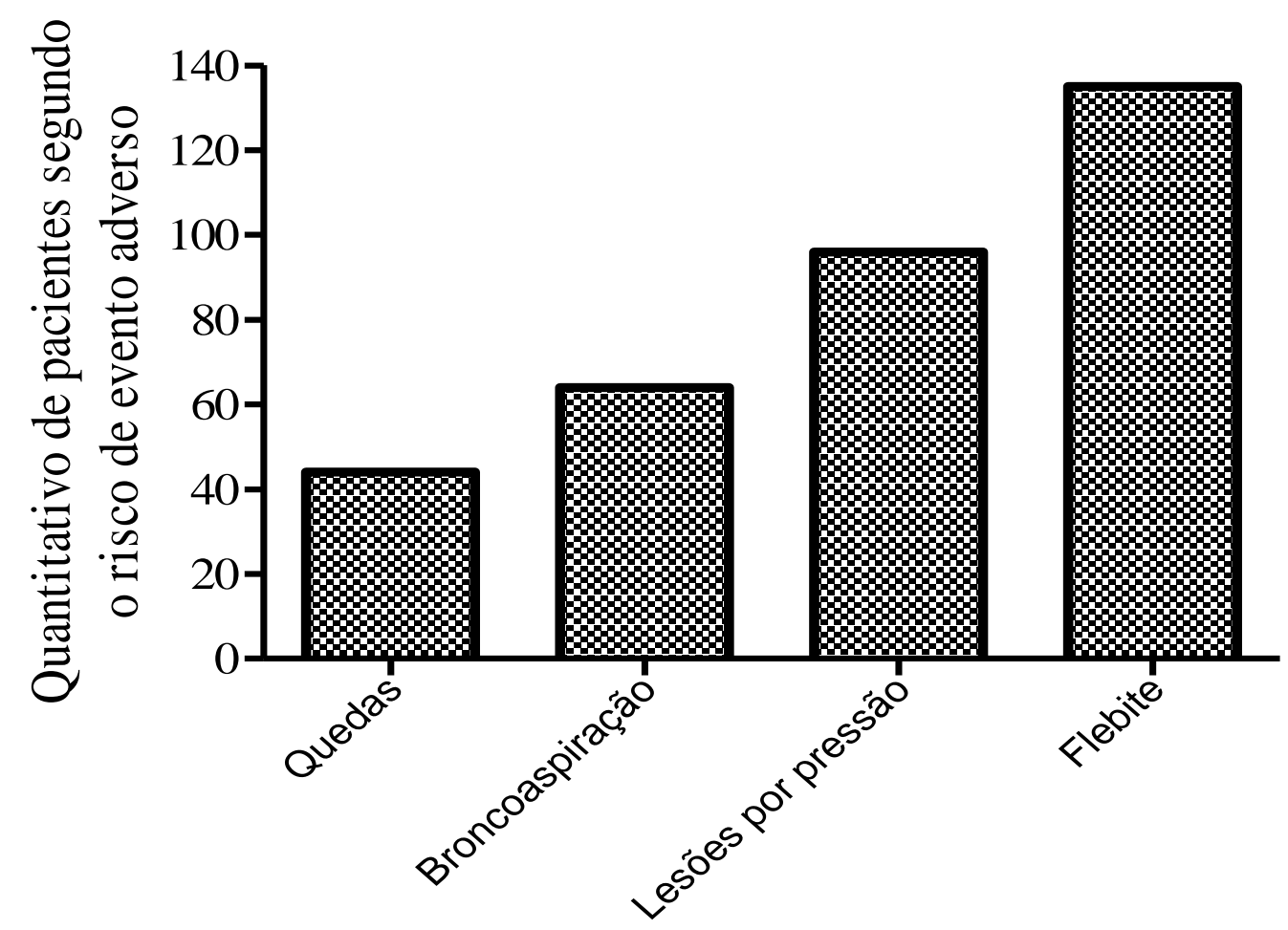

Gráfico 2. Quantitativo de pacientes segundo o risco de eventos adversos.

\section{DISCUSSÃO}

Os resultados encontrados neste estudo com respeito às variáveis demográficas e clínicas de pacientes internados na emergência corroboram evidências de outras investigações relacionadas a esse tipo de clientela.

Observou-se um predomínio do sexo masculino (65\%) na faixa etária de 50 a 69 anos (32,5\%). Os homens, por uma série de questões culturais e educacionais, só procuram o serviço de saúde quando perdem sua capacidade de trabalho ou estão em estado grave de saúde como infarto, acidente vascular cerebral, entre outros. Vale destacar ainda que a população idosa é caracterizada como vulnerável, elevando o grau de dependência dos cuidados, visto que o processo de envelhecimento está relacionado à perda da funcionalidade e ao aumento de comorbidades. ${ }^{10}$ 
Os riscos para eventos adversos são comumente associados ao erro humano individual, mas devem-se considerar como desencadeadores as condições de trabalho, os aspectos estruturais e a complexidade das atividades desenvolvidas. As situações que predispõem ao risco de eventos adversos incluem avanço tecnológico com deficiente aperfeiçoamento dos recursos humanos, desmotivação, falha na aplicação da sistematização da assistência de enfermagem (SAE), delegação de cuidados sem supervisão adequada e sobrecarga de serviço. ${ }^{11}$

Desse modo, compreender a relação entre riscos, características dos cuidados à saúde e aporte da rede hospitalar pode fornecer à enfermagem elementos importantes para a melhoria da assistência. Embora os riscos relacionados aos cuidados de enfermagem venham sendo abordados amplamente na literatura, torna-se importante conhecer o risco de eventos adversos para elaborar estratégias de prevenção. ${ }^{12}$

Neste estudo, foi possível identificar que a flebite representa o maior risco para o desenvolvimento de eventos adversos (82,8\%). No âmbito hospitalar, avaliada como um dos principais eventos adversos da terapia intravenosa, a flebite é uma das complicações mais frequentes e mais previsíveis. ${ }^{13}$ Ela decorre de uma inflamação formada na camada interna da parede vascular e segue associada a sinais e sintomas como dor, edema, vermelhidão, podendo levar, inclusive, à formação de cordão fibroso e, ainda, a aumento da temperatura local. Em casos infecciosos, está associada à presença de secreção purulenta em sítio de inserção do cateter. ${ }^{14}$

Complicações como essa podem ser atribuídas a uma série de fatores, como o tipo de cateter selecionado, o preparo do local de inserção, o tipo de infusão, a técnica de inserção, o tempo de permanência do cateter, o tipo de curativo e o local de inserção do cateter. ${ }^{15}$

Constatou-se também risco de $26,9 \%$ para o evento adverso queda. A queda de pacientes do leito ou da própria altura é outro evento adverso que necessita ser criteriosamente avaliado, podendo causar ferimentos e sequelas aos pacientes, prolongando o tempo e os custos da internação hospitalar, com consequente responsabilização legal da equipe de saúde e da instituição. Destaca-se que a prevenção de quedas é um indicador de resultado, além de ser um dos focos da ANVISA. ${ }^{16}$ Estudos apontam as quedas como um evento de alta incidência no ambiente hospitalar, com percentuais que variam de 1,1\% a $22 \%$, conforme a especificidade do paciente. ${ }^{17}$ Este incidente está diretamente relacionado à segurança do paciente e pode aumentar os dias de internação e interferir na recuperação do indivíduo. ${ }^{18}$

Já as quedas podem ser influenciadas por múltiplos fatores e acarretar consequências ao paciente como: danos, prolongamento do período de internação e aumento dos custos assistenciais. ${ }^{19} \mathrm{~A}$ avaliação do paciente e a identificação das características que podem aumentar a probabilidade de quedas torna-se fundamental para o planejamento de estratégias de prevenção efetivas. ${ }^{20}$ 
Outro evento adverso encontrado foi a broncoaspiração com risco potencial de $39,2 \%$ nos pacientes da unidade em estudo. Um estudo realizado em Fortaleza afirmou que o risco de broncoaspiração é real e altamente prevalente em unidades hospitalares. ${ }^{21} \mathrm{~A}$ broncoaspiração ocorre quando o paciente sofre inalação do conteúdo da boca ou do estômago para a laringe e trato respiratório inferior do pulmão. ${ }^{22}$ Os profissionais da equipe multidisciplinar (enfermeiros, médicos, fisioterapeutas e fonoaudiólogos) devem realizar ações preventivas visando à diminuição das ocorrências de broncoaspiração e à minimização dos riscos aos quais o paciente está exposto. ${ }^{21}$

Vale ainda ressaltar que quando não ocorre a realização de procedimentos como curativos e mudança de decúbito, o paciente é exposto a práticas não seguras de assistência, além de retardar a alta hospitalar, o retorno às atividades da vida diária e, consequentemente, ocasionar um aumento nos custos hospitalares, como é o caso da Lesão por pressão. ${ }^{23}$ Neste estudo, evidenciou-se que $58,8 \%$ dos pacientes apresentavam risco potencial para desenvolver este evento adverso.

Nessa concepção, a lesão por pressão (LP), reconhecida como um evento adverso, se ocorrer após a admissão do indivíduo no serviço de saúde, e como uma das cinco causas mais comuns de danos aos pacientes, apresenta-se como importante desafio. ${ }^{24}$ A LP é definida como um dano localizado na pele e/ou tecidos moles subjacentes, geralmente, sobre uma proeminência óssea, ou relacionada ao uso de dispositivo médico ou a outro artefato. A lesão pode se apresentar em pele íntegra ou como úlcera aberta, pode ser dolorosa, e ocorre como resultado da pressão intensa e/ou prolongada em combinação com o cisalhamento. A tolerância do tecido mole à pressão e ao cisalhamento pode também ser afetada pelo microclima, nutrição, perfusão, comorbidades e pela sua condição. ${ }^{25}$

Considerando a magnitude do problema da LP, a prevenção tem sido apontada como o melhor caminho para minimizar esse evento, com enfoque na utilização de diretrizes e protocolos clínicos. As diretrizes são declarações sistematicamente desenvolvidas de prática recomendada em uma área clínica específica, destinadas a fornecer orientações para os profissionais em sua prática, a partir de evidências atuais, visando, entre outros aspectos, reduzir a variabilidade nos cuidados, promover assistência segura e livre de danos e reduzir os custos com o cuidado. ${ }^{26}$

Cabe salientar que na ocorrência de eventos adversos, os mesmos devem ser notificados à Agência Nacional de Vigilância Sanitária (ANVISA) no sistema de notificações (NOTIVISA), que é um sistema informatizado onde profissionais, instituições e indivíduos podem notificar problemas relacionados ao uso de tecnologias, de processos assistenciais, produtos para a saúde, incidentes e eventos adversos. ${ }^{27}$

Destaca-se que a percepção diária de situações de risco colabora para o adequado gerenciamento do cuidado com enfoque na prevenção do erro e o estabelecimento da cultura de segurança na instituição. Os eventos adversos devem ser compreendidos em sua totalidade, considerando o que existe além de sua ocorrência, ou seja, sobrecargas de trabalho, a falta de conhecimento 
dos profissionais, falta de comunicação, precária infraestrutura institucional, dentre outros. ${ }^{23}$

O fortalecimento da cultura de segurança entre os profissionais destaca-se como um importante fator condicionador ao desenvolvimento institucional de estratégias para melhoria da qualidade e redução de incidentes. ${ }^{28}$

Cabe ressaltar que o desafio para o enfrentamento da redução dos riscos e dos danos na assistência à saúde dependerá da necessária mudança de cultura dos profissionais para a segurança, nos próximos anos, alinhada à política de segurança do paciente, instituída nacionalmente. Desta forma, investir na mudança de sistema, no aperfeiçoamento da equipe de saúde, na utilização de boas práticas e no aprimoramento das tecnologias e melhoria dos ambientes de trabalho constitui questões primordiais para o alcance dos melhores resultados e prevenir o desenvolvimento de eventos adversos. ${ }^{4}$

\section{CONCLUSÃO}

O desenvolvimento deste estudo permitiu concluir que a identificação de riscos é um importante passo para a prevenção de eventos adversos nos serviços hospitalares, sendo uma prática importante para qualificar a assistência prestada e impedir que o paciente desenvolva ou sofra algum dano durante o período intra-hospitalar

Ressalta-se que este estudo apresenta limitações por apresentar apenas o mapeamento de riscos para eventos adversos, uma vez que não foi analisada as notificações reais dos eventos adversos na unidade em estudo. Entretanto, comprova a necessidade de implementação de protocolos de identificação de riscos e desenvolvimento de ações preventivas para cada risco evidenciado, tendo em vista que tais medidas, além de aperfeiçoarem a assistência prestada, garantem ao paciente um atendimento humanizado e seguro.

O profissional da saúde, como ser humano, está sujeito a falhas e, assim sendo, evidencia-se a necessidade de notificar os eventos adversos para que se identifiquem as lacunas que precisam ser sanadas pelo serviço. Este aspecto é especialmente importante nas unidades de emergências, por atenderem pacientes de alto risco e em uso de recursos tecnológicos avançados que exigem profissionais competentes para seu manejo. Para tanto, ações de educação continuada com simulações de situações práticas é um caminho a ser cursado.

\section{REFERÊNCIAS}

1. Silva ACAB, Rosa DOS. Cultura de segurança do paciente em organização hospitalar. Cogitare Enferm. 2016 v. 21 n. esp: 01-10. Acesso em: 20 de janeiro de 2018. Disponível em: http://revistas.ufpr.br/cogitare/article/view/45583. 
2. Ministério da Saúde (BR). Portaria n. 529 de 1ํ de abril de 2013: institui o Programa Nacional de Segurança do Paciente (PNSP). Brasília (DF): MS; 2013. Acesso em: 20 de janeiro de 2018. Disponível em:

http://bvsms.saude.gov.br/bvs/saudelegis/gm/2013/prt0529 0104 2013.ht $\underline{\mathrm{ml}}$.

3. BRASIL. Agência Nacional de Vigilância Sanitária. Gestão de Riscos e Investigação de Eventos Adversos Relacionados à Assistência à Saúde. Brasília: Anvisa, 2017. Acesso em: 20 de janeiro de 2018. Disponível em: http://portal.anvisa.gov.br/documents/33852/3507912/Caderno+7++Gest\%C 3\%A3o+de+Riscos+e+Investiga\%C3\%A7\%C3\%A30+de+Eventos+Adverso s+Relacionados+\%C3\%A0+Assist\%C3\%AAncia+\%C3\%A0+Sa\%C3\%BAde /6fa4fa91-c652-4b8b-b56e-fe466616bd57.

4. BRASIL. Agência Nacional de Vigilância Sanitária. Assistência Segura: Uma Reflexão Teórica Aplicada a Prática. 2013. Acesso em: 21 de janeiro de 2018. Disponível em:

http://www20.anvisa.gov.br/segurancadopaciente/images/documentos/livros Livrol- Assistencia Segura.pdf.

5. Wachter, R. Compreendendo a Segurança do Paciente. 2a Ed. Porto Alegre: AMGH, 2013. 478p.

6. BRASIL. Agência Nacional de Vigilância Sanitária Implantação do Núcleo de Segurança do Paciente em Serviços de Saúde - Série Segurança do Paciente e Qualidade em Serviços de Saúde/Agência Nacional de Vigilância Sanitária- Brasília: Anvisa, 2014.

http://www.saude.pr.gov.br/arquivos/File/OSEGURANCA DO PACIENTE/m odulo6.pdf.

7. Silva-Batalha EMS, Melleiro MM. Cultura de segurança do paciente em um hospital de ensino: diferenças de percepção existentes nos diferentes cenários dessa instituição. Texto Contexto Enferm, Florianópolis, 2015 AbrJun; 24(2): 432-41. Acesso em: 21 de janeiro de 2018. Disponível em: http://www.scielo.br/pdf/tce/v24n2/pt 0104-0707-tce-24-02-00432.pdf.

8. Alves RS, Silveira EMC. A presença de idosos num serviço de emergência: campo de saúde ou campo de guerra? Revista Kairós Gerontologia, 17(1), pp.297-319. Acesso em: 25 de janeiro de 2018. Disponível em: https://revistas.pucsp.br/index.php/kairos/article/view/21331/15623

9. Silva JDS, Cortez LER. Principais causas externas dos idosos atendidos nas unidades de urgência e emergência. Vol.23, n.3, pp.38-43 (Jul - Set 2015). Acesso em: 25 de janeiro de 2018. Disponível em: https://www.mastereditora.com.br/periodico/20150902 110535.pdf.

10. Girondi JBR, Nothaft SCS, Santos SMA, Oliveira F, Sebold LF, Kempfer SS. Estudo do perfil de morbimortalidade entre idosos. Rev Enferm UFSM. 2013;3(2):197-204. 
11. Beccaria LM, Pereira RAM, Contrin LM, Lobo SMA, Trajano DHL. Eventos adversos na assistência de enfermagem em uma unidade de terapia intensiva. Rev. bras. ter. intensiva. 2009; 21(3): 276-82.

12. Oliveira RM, Leitão IMTA, Silva LMS, Figueiredo SV, Sampaio RL, Gondim MM. Estratégias para promover segurança do paciente: da identificação dos riscos às práticas baseadas em evidências. Esc Anna Nery 2014;18(1):122129. Acesso em: 25 de janeiro de 2018. Disponível em: http://www.scielo.br/pdf/ean/v18n1/1414-8145-ean-18-01-0122.pdf

13. Yassuko ACM, Silva VGLG, Aparecido BLJ, Camboin MV, Willamowius VD, Misue ML. Avaliação de cuidados na terapia intravenosa: desafio para a qualidade na enfermagem. Escola Anna Nery rev enferm. 2013;17(1):11-6.

14. Magerote NP, Lima MHM, Silva JB, Correia MDL, Secoli SR. Associação entre flebite e retirada de cateteres intravenosos periféricos. Texto contexto enferm. 2011;20(3):486-92. Acesso em: 25 de janeiro de 2018. Disponível em: http://www.scielo.br/scielo.php?script=sci arttext\&pid=S0104$\underline{07072011000300009}$

15. Callaghan S, Copnell B, Johnston L. Comparison of two methods of peripheral intravenous cannula securement in the pediatric setting. J Infus Nurs 2002; 25(4):256-64.

16. BRASIL. Agência Nacional de Vigilância Sanitária. Resolução da Diretoria Colegiada - RDC no. 36, de 25 de julho de 2013. Institui ações para a segurança do paciente em serviços de saúde e dá outras providências. Diário Oficial da União 2013. Acesso em: 25 de janeiro de 2018. Disponível em: http://bvsms.saude.gov.br/ bvs/saudelegis/anvisa/2013/rdc0036 2507 2013.html.

17. Vieira ER, Berean C, Paches D, Caveny P, Yuen D, Ballash L, et al. Reducing falls among geriatric rehabilitation patients: a controlled clinical trial. Clin Rehabil. [Internet]. 2013. Acesso em: 29 de janeiro de 2018. Disponível em: https://www.ncbi.nlm.nih.gov/pubmed/22952303

18. Abreu C, Mendes A, Monteiro J, Santos FR. Falls in hospital settings: a longitudinal study. Rev. Latino-Am. Enfermagem. [Internet]. May-June 2012. Acesso em: 29 de janeiro de 2018. Disponível em: http://www.scielo.br/pdf/rlae/v20n3/a23v20n3.pdf.

19. Costa SGRF, Monteiro DR, Hemesath MP, Almeida MA. Caracterização das quedas do leito sofridas por pacientes internados em um hospital universitário. Rev Gaúcha Enferm. [Internet]. Dez. 2011 Acesso em: 29 de janeiro de 2018. Disponível em: http://www.scielo.br/pdf/rgenf/v32n4/v32n4a06.

1. 20. Luzia MF, Victor MAG, Lucena AF. Nursing Diagnosis Risk for falls: prevalence and clinical profile of hospitalized patients. Rev. Latino-Am. Enfermagem [Internet]. Mar.- Apr. 2014. Acesso em: 29 de janeiro de 2018. 
Disponível em: http://www.scielo.br/pdf/rlae/v22n2/0104-1169- rlae-22-0200262.pdf.

20. Almeida AEM, Alcântara ACC, Lima FAM, Rocha HAL, Cremonin Junior JR, Costa HJM. Prevalência de risco moderado e alto de aspiração em pacientes hospitalizados e custo-efetividade da aplicação de protocolo preventivo. J Bras Econ Saúde 2016;8(3): 216-220.

21. Marik PE. Pulmonary aspiration syndromes. Curr Opin Pulm Med. 2011;17(3):148-54.

22. Duarte SCM, Stipp MAC, Silva MM, Oliveira FT. Eventos adversos e segurança na assistência de enfermagem. Rev Bras Enferm. 2015 janfev;68(1):144-54. Acesso em: 29 de janeiro de 2018. Disponível em: http://www.scielo.br/pdf/reben/v68n1/0034-7167-reben-68-01-0144.pdf.

23. Elliott R, McKinley S, Fox V. Quality improvement program to reduce the prevalence of pressure ulcers in an intensive care unit. Am J Crit Care [Internet]. 2008. Acesso em: 29 de janeiro de 2018. Disponível em: http://ajcc.aacnjournals.org/content/17/4/328.full.pdf.

24. National Pressure Ulcer Advisory Panel. National Pressure Ulcer Advisory Panel (NPUAP) announces a change in terminology from pressure ulcer to pressure injury and updates the stages of pressure injury. [Internet]. 2016 Abr. Acesso em: 29 de janeiro de 2018. Disponível em: http://www.npuap.org/national-pressure-ulcer-advisory-panelnpuapannounces-a-change-in-terminology-from-pressure-ulcer-topressureinjury-and-updates-the-stages-of-pressure-injury/.

25. Registered Nurses Association of Ontario. Toolkit: Implementation of Best Practice Guidelines. Guideline supplement. Toronto (CAN): RN Ass of Ontario; 2012 Set. Acesso em: 29 de janeiro de 2018. Disponível em: http://rnao.ca/sites/rnao-ca/files/RNAO ToolKit 2012 rev4 FA.pdf.

26. Bezerra ALQ, Silva AEBC, Branquinho NCSS, Paranaguá TTB. Análise de queixas técnicas e eventos adversos notificados em um hospital sentinela. Rev Enferm UERJ. 2009;17(4):467-72. Acesso em: 29 de janeiro de 2018. Disponível em: http://www.facenf.uerj.br/v17n4/v17n4a02.pdf.

27. Marchon SG, Junior WVM, Pavão ALB. Características dos eventos adversos na atenção primária à saúde no Brasil. Cad. Saúde Pública, Rio de Janeiro, 31(11):2313-2330, nov, 2015. Acesso em: 29 de janeiro de 2018. Disponível em: http://www.scielo.br/pdf/csp/v31n11/0102-311X-csp31-11-2313.pdf.

Recebido: 30 de janeiro de 2018. Publicado: 05 de março de 2018 
Correspondência: Maria Girlane Sousa Albuquerque Brandão. E-mail: girlane.albuquerque@yahoo.com.br

Conflito de Interesses: os autores declararam não haver conflito de interesses.

(C) This is an Open Access article distributed under the terms of the Creative Commons Attribution License, which permits unrestricted use, distribution, and reproduction in any medium, provided the original work is properly cited 